\title{
Isolement de souches de mycobactéries pathogènes chez des bovins abattus à l'abattoir de Bobo-Dioulasso, Burkina Faso
}

\author{
A. Delafosse ${ }^{1}$, A. Traore ${ }^{2}$, B. Kone ${ }^{3}$
}

DELAFOSSE A., TRAORE A., KONE B. Isolement de souches de mycobactéries pathogènes chez des bovins abattus à l'abattoir de BoboDioulasso, Burkina Faso. Revue Elev. Méd vét. Pays trop., 1995, 48 (4) : 301-306

La tuberculose bovine est le premier motif de saisie à l'abattoir de Bobo-Dioulasso. Ce travail a consisté à confirmer, par des méthodes de laboratoire (examen direct après coloration de Ziehl-Nielsen et isolement de souches de mycobactéries après culture sur milieu de Loewenstein-Jensen), le diagnostic nécropsique effectué à l'abattoir. Pendant la durée de cette étude, 39 souches de mycobactéries pathogènes ( 38 souches de Mycobacterium bovis et 1 souche de $M$. tubercu. losis) ont pu être isolées à partir de $\mathbf{1 0 0}$ prélèvements suspects. Aucune mycobactérie évoquant $M$. farcinogenes n'a pu être observée à l'examen direct. Le diagnostic différentiel entre la tuberculose bovine et le farcin a pu donc être posé. Ces résultats confirment le bien fondé des saisies pour tuberculose effectuées à l'abattoir. La majorité des bovins provenant de villages situés à proximité de Bobo-Dioulasso, il est probable que cette maladie sévisse à l'état enzootique dans la région, avec un taux d'infection chez les animaux certainement élevé.

Mots-clés : Bovin - Tuberculose - Mycobacterium - Agent pathogène Lésion - Nécropsie - Abattoir - Burkina Faso.

\section{INTRODUCTION}

L'abattoir de Bobo-Dioulasso (Burkina Faso) est le deuxième du pays par son importance. Ainsi, du 1er avril 1992 au 1er avril 1993, 73 bovins y ont été abattus en moyenne chaque jour. L'analyse du registre des services sanitaires $(2,11)$ montre que, pendant cette période, le premier motif de saisie des viandes bovines est la tuberculose. En moyenne, 10 saisies partielles par jour et 1 à 2 saisies totales par mois sont effectuées pour ce motif.

Le diagnostic clinique de la tuberculose effectué à l'abattoir est-il fiable ? Ne peut-il y avoir confusion entre la tuberculose vraie et le farcin du boeuf (à Mycobacterium farcinogenes) très comparable du point de vue symptomatologique?

Une série d'enquêtes tuberculiniques, utilisant l'intradermo-réaction comparative, a été effectuée entre 1967 et

1. CIRDES, 01 BP 454, Bobo-Dioulasso 01, Burkina Faso.

2. Ecole nationale vétérinaire d'Alfort, 7 avenue du Général de Gaulle, 94704 Maisons-Alfort Cedex, France.

3. Service provincial des Ressources animales du Houet, BoboDioulasso, Burkina Faso.

Reçu le 15.9.95, accepté le 5.2.96.
$1976(5,12,16)$ dans différentes régions du Burkina Faso (alors Haute Volta).

Dans la zone sahélienne du pays, 3 à 7 p. 100 des bovins testés à la tuberculine humano-bovine réagissaient. L'importance de la tuberculose bovine était très variable d'un village à l'autre (jusqu'à 17 p. 100 d'animaux positifs). Les animaux âgés étaient les plus touchés. A Gaoua (ville située au sud-ouest du Burkina Faso), 6 à 7 p. 100 des bovins réagissaient à la tuberculine humano-bovine. Par ailleurs, sur 209 souches de mycobactéries isolées chez les bovins à partir des abattoirs de la région de Bobo-Dioulasso entre 1965 et 1968 , 192 (92 p. 100) étaient Mycobacterium bovis, 7 (3,4 p. 100) $M$. tuberculosis et $10(4,6$ p. 100) des mycobactéries atypiques (6).

Ces résultats tendent à montrer l'existence, à l'époque, de tuberculose bovine à l'état enzootique au Burkina Faso, la région nord du pays étant la plus touchée. Les seules données épidémiologiques récentes sont fournies par les rapports statistiques publiés chaque année par le Ministère de l'Agriculture et de l'Elevage. Ces statistiques se basent sur les saisies effectuées dans les abattoirs du pays.

Ce travail a pour objectif d'évaluer la prévalence de la tuberculose vraie dans la population des bovins traités par l'abattoir de Bobo-Dioulasso du 21 juin au 28 juillet 1994. II s'agit de confirmer le diagnostic de tuberculose posé à l'abattoir à l'aide de techniques de laboratoire. L'examen bactériologique, après coloration au Ziehl-Nielsen, et la mise en culture sur milieu de Loewenstein-Jensen ont été utilisés.

\section{MATERIEL et METHODES}

La population étudiée est constituée par l'ensemble des bovins traités à l'abattoir de Bobo-Dioulasso du 21 juin au 28 juillet 1994. Le recueil des prélèvements a été effectué par un étudiant de l'Ecole vétérinaire de Maisons-Alfort (14).

Pendant la période de l'étude, l'ensemble des organes saisis pour cause de tuberculose a été prélevé chaque jour dans des boites stériles.

A partir de chaque prélèvement, un étalement a été coloré à froid avec le colorant de Ziehi-Nielsen. Chaque lame 
a été lue et interprétée par deux personnes habituées à ce type d'examen.

La richesse des étalements en bacilles alcoolo-acidorésistants (BAAR) est appréciée en utilisant l'échelle suivante :

$\begin{array}{ll}\text { P0 } & \text { lame négative } \\ \text { P1 } & \text { 1 BAAR pour } 100 \text { champs } \\ \text { P2 } & \text { 2 BAAR pour } 100 \text { champs } \\ \text { P3 } & \text { 3 BAAR pour } 100 \text { champs } \\ \text { P4 } & \text { 4 BAAR pour } 100 \text { champs } \\ \text { P5 } & 5 \text { BAAR pour } 100 \text { champs } \\ \text { P6 } & \text { 6 à } 24 \text { BAAR pour } 100 \text { champs } \\ \text { P7 } & \text { 25 à } 40 \text { BAAR pour } 100 \text { champs } \\ \text { P8 } & \text { 41 à } 80 \text { BAAR pour } 100 \text { champs } \\ \text { P9 } & \text { nombreux BAAR par champ. }\end{array}$

Pour chaque prélèvement, des procédés de décontamination (traitement par l'acide sulfurique puis par la soude) sont réalisés avant ensemencement sur le milieu de Loewenstein-Jensen. La région d'origine des animaux ayant fait l'objet de saisies pour tuberculose a également été relevée.

\section{RESULTATS}

Sur les 2700 bovins abattus pendant la durée de ce travail, il y a eu 93 saisies partielles et 7 saisies totales pour tuberculose (soit 3,7 p. 100 d'animaux suspects de tuberculose à l'issue du diagnostic clinique).

\section{Localisation des lésions}

La répartition des cas selon la localisation des lésions fait apparaître une prédominance des formes pulmonaires devant les formes mammaires et rénales (tableau I).

\section{TABLEAUU I}

Répartition des prélèvements en fonction des organes atteints

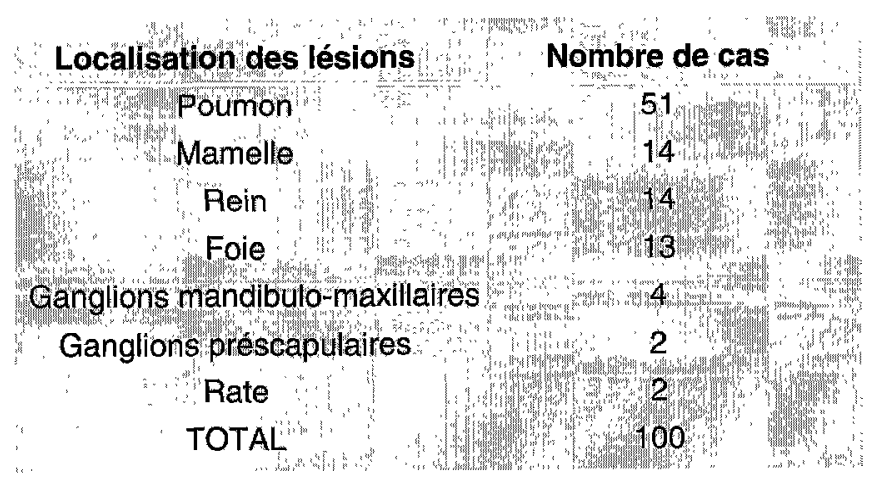

\section{Examen direct après coloration de Ziehl-Nielsen}

Des BAAR ont été retrouvés dans 54 des 100 prélèvements effectués. Les bacilles filiformes, «en perruque», caractéristiques de $M$. farcinogenes, n'ont jamais été rencontrés.

Les résultats exprimés en fonction de la richesse des éléments en BAAR et de la nature de l'organe prélevé (tableau II) montrent que la moitié des lames positives ne compte que 1 à 4 bacilles pour 100 champs.

\section{TABLEAU II}

Résultats de l'examen direct en fonction de la nature de l'organe prélevé

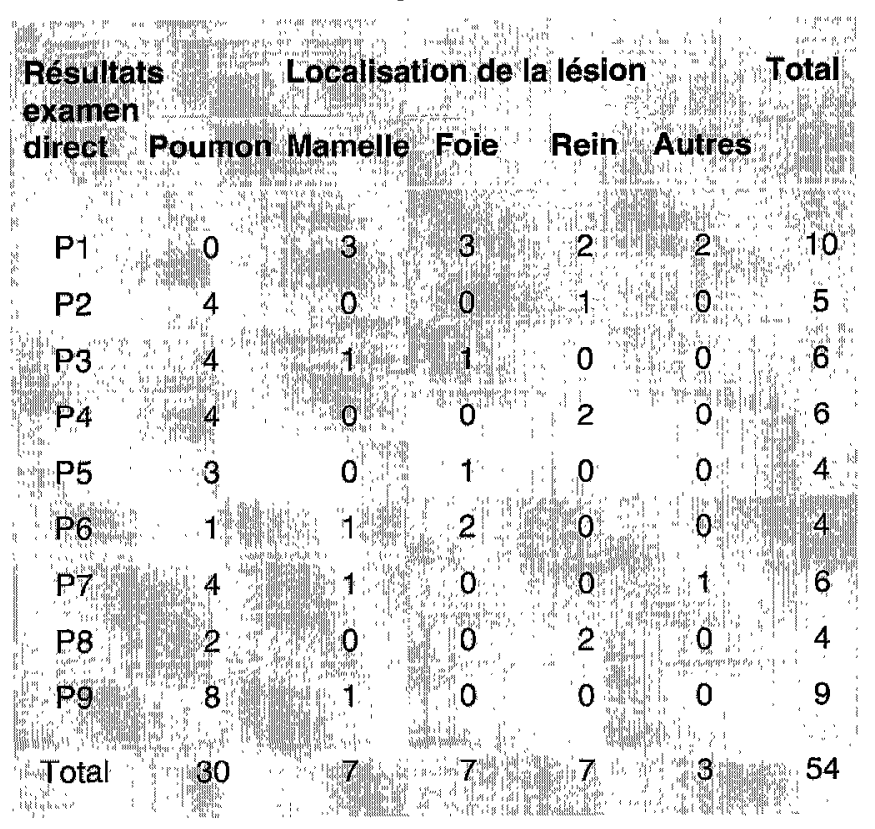

Sur les 9 étalements les plus riches en BAAR (nombreux bacilles par champ), 8 provenaient de lésions pulmonaires (tableau II) et une d'une lésion de la mamelle. Ces différences entre organes ne sont pas significatives (tableau III) (test du $\chi^{2}$ corrigé de Yates ; $\chi^{2}=3,37, \mathrm{NS}$ ).

\section{TABLEAU III}

Résultats de l'examen direct en fonction de la localisation des lésions

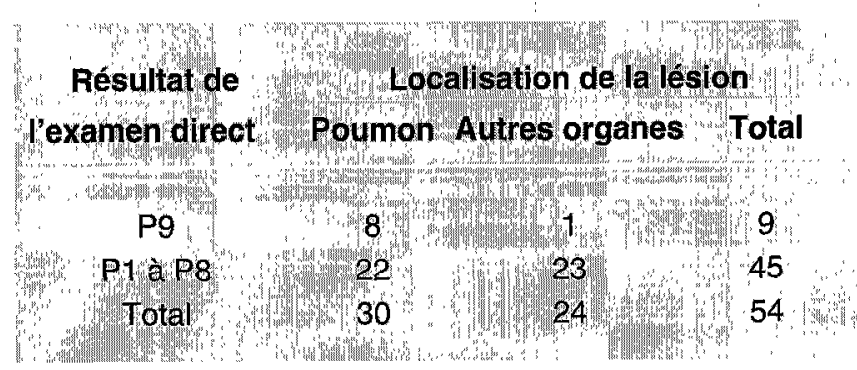




\section{Cultures sur milieu de Loewenstein-Jensen}

Sur 100 prélèvements ensemencés, 86 ont poussé et 14 se sont révélés non interprétables, les cultures ayant été contaminées.

L'identification des mycobactéries a pu être entreprise sur 39 des 86 cultures et 47 ont donné des résultats négatifs. Les résultats montrent la prédominance de $M$. bovis (38 isolements pour 39 cultures positives).

Aucune souche de $M$. avium n'a été isolée. Une souche de $M$. tuberculosis a pu être isolée d'un prélèvement par ailleurs négatif à l'examen direct (tableau IV).

Sur 39 cultures, 27 cultures positives (soit 69,2 p. 100) proviennent de lésions pulmonaires et $3(7,7$ p. 100) de lésions mammaires.

Sur 40 prélèvements négatifs à l'examen direct, on a pu isoler dans 8 cas (soit 20 p. 100) des mycobactéries pathogènes. Lorsque le nombre de BAAR pour 100 champs est faible ( $\mathrm{P} 1$ à $\mathrm{P} 5$ selon le barème retenu), on isole des mycobactéries pathogènes dans 50 p. 100 des cas. Ce taux passe à 82 p. 100 lorsque le résultat de l'examen direct est compris entre P6 et P8, et à $100 \mathrm{p}$. 100 quand le prélèvement comprend de nombreux BAAR par champ (P9) (tableau V).

\section{TABLEAU V}

Résultats comparés des examens directs après coloration et des cultures sur milieu de Loewenstein-Jensen

\begin{tabular}{|c|c|c|c|c|c|}
\hline Culture & , & Ex & amen dir & & \\
\hline 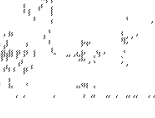 & négatif & P1 à P5 & P6 à P8 & Pg & Total \\
\hline Positive & 8 & 13 & 9 & 9 & 39 \\
\hline Négative & 32 & 13 & 2 & 0 & 47 \\
\hline Total & 40 & 26 & 11 & 9 & 86 \\
\hline
\end{tabular}

Les cultures contaminées n'ont pas été retenues. Le test du $\chi^{2}$ de tendance est significatif $\left(\chi^{2}\right.$ tendance $=21,8,1$ ddl $; \mathrm{p}<0,001)$.

TABLEAU IV

Identification des mycobactéries à l'aide de la mise en culture des prélèvements

\section{Résultat \\ dela" \\ culture}

\section{M. bovis \\ M. bovis \\ $M$ bovis}

M. bovis

M. bovis

M. bovis

$M$ bovis

M. bovis

M. bovis

M. bovis

$M$ bovis

M bovis

M. bovis

M. bovis

$M$ bovis

M bovis

M. bovis

M. bovis

$M$ bovis

M. bovis

M. bovis

M. bovis

M. bovis

M. bovis

M. bovis

$M$. bovis

M. bovis

$M$. bovis

$M$. bovis

M. bovis

M. bovis

M. bovis

M. bovis

M. bovis

$M$. bovis

$M$. bovis

M. bovis

M. bovis

M. tuberculosis

\section{Résultat de l'examen direct}

P1

$\mathrm{P} 2$

P2

P3

P3

P3

P4

$\mathrm{P} 4$

P4

P5

P5

P5

P5

P6

P6

P7

P7

P7

P7

P7

P8

P8

P9

P9

P9

P9

P9

P9

P9

P9

$$
\text { P9 }
$$

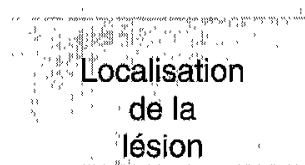

Mamelle

Poumon

Poumon

Poumon

Poumon

Poumon

Poumon

Rein

Poumon

Poumon

Poumon

Poumon

Foie

Foie

Poumon

Ganglion

mandibulo-maxillaire

Poumon

Poumon

Poumon

Mamelle

Poumon

Poumon

Poumon

Poumon

Mamelle

Poumon

Poumon

Poumon

Poumon

Poumon

Poumon

Négatif

Poumon

Négatif

Poumon

Négatif

Rate

Négatif

Rein

Négatif

Foie

Négatif

Foie

Négatif

Foie

Négatif

Poumon 
Par ailleurs, on note une différence en fonction de la localisation des lésions. Ainsi, lorsque l'organe prélevé est le poumon, le pourcentage de cultures positives est significativement plus élevé pour les prélèvements contenant un nombre faible ( $\mathrm{P} 1$ à $\mathrm{P} 5)$ ou moyen ( $\mathrm{P} 6$ à $\mathrm{P} 8)$ de BAAR à l'examen direct (tableau VI).

\section{TABLEAU VI}

Résultats des cultures sur milieu de Loewenstein-Jensen pour des prélèvements contenant un nombre faible ou moyen de BAAR à l'examen direct

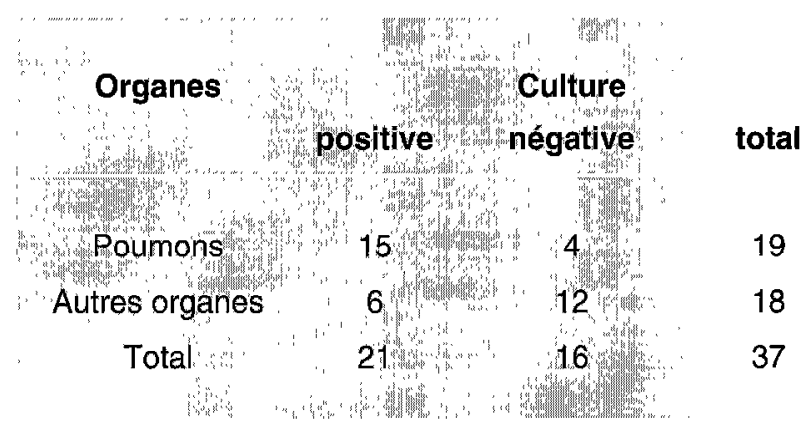

Comparaison en fonction des organes prélevés

Les différences observées sont significatives au test du $\chi^{2}\left(\chi^{2}\right.$ calculé $\left.=7,77 ; p<0,01\right)$.

En prenant la culture sur milieu de Loewenstein-Jensen comme technique de référence, nous pouvons calculer la sensibilité et la spécificité de l'examen direct après coloration de Ziehl-Nielsen (tableaux VII et VIII).

\section{TABLEAU VII}

Sensibilité et spécificité de l'examen direct pour les prélèvements issus de lésions pulmonaires

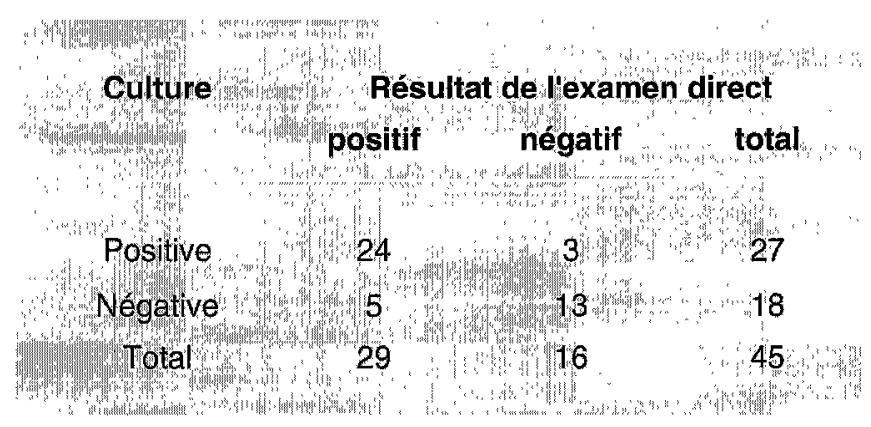

Sensibilité (Sn) - vrais positifs (VP)/(vrais positifs (VP) + faux négatifs (FN)) = $24 / 27=0,89$

Spécificité (Sp) - vrais négatifs (VN)/(vrais négatifs (VN) + faux positifs (FP)) $=13 / 18=0,72$

\section{TabLEaU VIII}

Sensibilité et spécificité de l'examen direct pour les prélèvements issus d'autres organes que les poumons

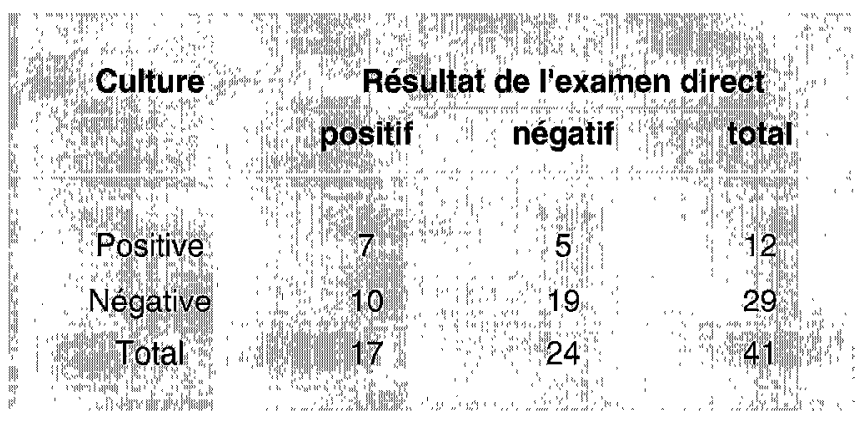

$S n=7 / 12=0,58 \quad S p=19 / 29=0,65$

Les animaux ayant fait l'objet de saisies pour tuberculose provenaient dans 87 p. 100 des cas, du quart sud-ouest du pays et souvent de villages situés à proximité de Bobo-Dioulasso (72 p. 100 des cas) ; 13 p. 100 avaient été achetés au nord, dans la zone sahélienne du pays.

\section{DISCUSSION}

Cette étude permet de démontrer l'existence de tuberculose dans la population de bovins, abattus à Bobo-Dioulasso du 21 juin au 28 juillet 1994.

Le diagnostic différentiel avec le farcin du boeuf a pu être fait, aucun BAAR de forme filamenteuse, caractéristique de Mycobacterium farcinogenes n'ayant été observé. Le farcin du boeuf ne semble pas être une pathologie fréquente au Burkina Faso, cette constatation ayant déjà été faite en 1969 (7).

Ce travail permet également de comparer deux techniques de diagnostic : le simple examen microscopique après une coloration de Ziehl-Nielsen et la mise en culture sur le milieu de Loewenstein-Jensen. II apparaît que plus le nombre de BAAR observés à l'examen direct est grand, plus la probabilité d'isoler une mycobactérie pathogène sur le même prélèvement est importante.

Certains auteurs (9) évoquent la présence accidentelle de mycobactéries saprophytes dans le pus. Ces germes, en petite quantité car n'étant pas dans un milieu permettant leur multiplication, pourraient expliquer l'existence de quelques BAAR dans certains prélèvements.

La présence de glycérine dans le milieu de LoewensteinJensen pourrait aussi inhiber le développement de $M$. bovis (15). Dès lors, les lésions pauvres en bacilles, comme c'est souvent le cas dans l'espèce bovine (10), ne permettraient pas d'isoler $M$. bovis. 
Un résultat négatif sur un seul prélèvement ne peut permettre d'éliminer la tuberculose car l'émission de mycobactéries est souvent discontinue (10). Le nombre de cas de tuberculose vraie enregistré au cours de notre étude est donc certainement sous-évalué.

Les spécificités et sensibilités de l'examen direct calculées dans notre étude font apparaître une différence en fonction de la nature de l'organe prélevé. Ainsi la sensibilité du test pour les lésions pulmonaires est de 0,89 contre seulement 0,58 pour les lésions des autres organes. Dans les deux cas, les spécificités obtenues sont faibles (respectivement 0,72 et 0,65 ), la valeur la plus élevée étant obtenue avec les lésions pulmonaires.

Ces résultats indiquent qu'un simple examen microscopique après une coloration de Ziehl-Nielsen permet de mesurer une prévalence apparente très éloignée de la prévalence réelle, notamment en ce qui concerne les formes de tuberculose extra-pulmonaires.

Le cas de tuberculose respiratoire à $M$. tuberculosis peut être lié à une contamination humaine, l'animal ayant été infecté au contact d'un éleveur malade. $M$. tuberculosis a été isolé dans 3,4 p. 100 des cas lors d'une étude réalisée entre 1965 et 1968 à Bobo-Dioulasso sur 209 prélèvements d'origine bovine suspects (6). Sur 610 souches isolées en France chez les bovins entre 1983 et 1992 (13), il n'y avait que 0,5 p. 100 de M. tuberculosis.

Au Burkina Faso, le bacille humain semble donc jouer un rôle non négligeable dans l'épidémiologie de la tuberculose bovine, ce qui pourrait être lié à la forte incidence de la tuberculose humaine dans le pays. Même si $M$. tuberculosis n'induit pas de tuberculose progressive chez les bovins, il peut survivre un certain temps dans leurs tissus $(6,10)$. Les bovins pourraient donc jouer un rôle de réservoir dans la tuberculose humaine à $M$. tuberculosis, les éleveurs étant particulièrement exposés à ce type de contamination.

La population étudiée ici ne peut être considérée comme représentative du troupeau bovin du Burkina Faso. Les résultats obtenus ne sont donc pas extrapolables à celuici. Les seules conclusions valides concernent la population des bovins abattus à Bobo-Dioulasso pendant la période de l'étude. Ces résultats confirment le bien-fondé des saisies pour tuberculose effectuées à l'abattoir. Par ailleurs, la situation épidémiologique actuelle de la tuberculose bovine au Burkina Faso étant très mal connue, la forte prévalence enregistrée à l'abattoir de Bobo-Dioulasso permet de penser qu'une forte proportion des cheptels bovins du pays est infectée.

Les pertes économiques dues à la tuberculose bovine avant l'application des mesures de lutte en France ont été estimées à 3 p. 100 de la production bovine (9). De plus, l'impact de cette maladie sur la santé publique est important, puisqu'il s'agit d'une zoonose majeure. Ainsi, au Pérou, une étude portant sur 853 souches responsables de tuberculose pulmonaire de l'homme en identifia
$38(4,45$ p. 100) comme étant $M$. bovis (3). On estime qu'avant que la tuberculose bovine ne soit contrôlée, 50 p. 100 des adénites cervicales humaines dans les îles britanniques étaient dues à $M$. bovis (1).

On estime que 5 à 7 p. 100 des vaches positives à l'intradermo-réaction (IDR) excrètent du bacille dans le lait en l'absence de lésion mammaire apparente (9). Cela explique le nombre important d'enfants atteints de tuberculose à $M$. bovis en Amérique latine (1), ceux-ci étant de grands consommateurs de lait cru.

Une étude a été menée de 1974 à 1977 (12) dans la zone sahélienne du Burkina Faso, peuplée majoritairement d'éleveurs consommateurs de lait cru, pour mettre en évidence des cas de tuberculose humaine à $M$. bovis. L'étude bactériologique a porté sur 55 souches dont une seule avait les caractéristiques de $M$. bovis. Cette faible prévalence apparente des infections à $M$. bovis, dans une zone où les populations sont très exposées au risque, pourrait s'expliquer par l'absence de contagion interhumaine (8). Il est probable que la prévalence réelle de la tuberculose humaine à $M$. bovis soit sous-estimée car les malades atteints de formes pulmonaires éliminent moins de bacilles dans leurs crachats que ceux infectés par M. tuberculosis (8). Par ailleurs, le risque de tuberculose secondaire est plus faible chez les sujets infectés par $M$. bovis (10) et les formes extra-pulmonaires ne sont que très rarement diagnostiquées.

Une enquête épidémiologique descriptive, utilisant l'intradermo-réaction menée sur un échantillon représentatif du troupeau bovin du Burkina Faso, serait un préalable indispensable à une bonne connaissance de l'épidémiologie de la tuberculose dans le pays.

\section{Remerciements}

Nous tenons à remercier le Dr S.M. Touré, Directeur Général du CIRDES, et le Dr S. Ledru, Laboratoire de Bactériologie du Centre Muraz, Bobo Dioulasso. La mise en culture des échantillons a été effectuée au Centre Muraz.

\section{Bibliographie}

1. ACHA P.N., SLYFRES B., 1989. Loonoses et maladies transmissibles communes à l'homme et aux animaux, 2ème ed. Paris, France, Office International des Epizooties, $1038 \mathrm{p}$.

2. DELAFOSSE A., 1993-1994. Unité d'Epidémiologie et de Biotechnologies appliquées. Bobo-Dioulasso, Burkina Faso, CIRDES, 46 p. (Rapport d'activités)

3. FERNANDEZ SALAZAR M., GOMEZ PANDO V., DOMINGUEZ PARCDES L., 1983. M. bovis en la patologica humana en cl Peru. Bol. Inf. Colegio Med, vet. Peru. 14 : 16-18.

4. FRANCIS J., 1958. Tubcrculosis in animals and man. A study in comparative pathology. Londres, United Kingdom, Cassel. 


\section{A. Delafosse A. Traore B. Kone}

5. GIDEL R., ALBERT J.P., 1967. Enquête sur la tuberculose bovine au moyen de tests tuberculiniques dans la région de Dori (Haute-Volta) du 14/11 au 03/12/67. Bobo Dioulasso, Haute-Volta, OCCGE. (Doc. technique $\mathrm{N}^{\circ} 72$ (Zoonoses)

6. GIDEL R., ALBERT J.P., LEFEVRE M., MENARD M., RETIF M., 1969. Les mycobactéries d'origine animale isolées au Centre Muraz de 1965 à 1968. Techniques d'isolement et d'identification. Résultats. Revue Elev. Méd. vét. Pays trop., 22 (4) : 495-508.

\%. GIDEL R., ALBERT J.P., RETIF M., 1969. Enquête sur la tuberculose bovine au moyen de tests tuberculiniques dans diverses régions d'Afrique occidentale. Revue Elev. Méd. vét. Pays trop., 22 (3) : 337-355.

8. GRIFFITHS A.S., 1937. Bovine tuberculosis in man. Tubercle, 18: 528543.

9. ECOLES NATIONALES VETERINAIRES FRANCAISES, CHAIRES DES MALADIES CONTAGIEUSES, 1990. La tuberculose. Lyon, France, Rhône-Mérieux, 152 p.

10. MAGNUS K., 1966. Epidemiological basis of tuberculosis eradication. Risks of pulmonary infection. Bull. OMS, $35: 483-508$.

DELAFOSSE A. , TRAORE A. , KONE B. Isolation of pathogenic Mycobacterium strains in cattle slaughtered in Bobo-Dioulasso, Burkina Faso. Revue Elev. Méd vét. Pays trop., 1995, 48 (4) : 301-306

The major cause of meat rejection at the slaughterhouse of BoboDioulasso is bovine tuberculosis. The objective of this work was to confirm the postmortem diagnosis pronounced at the slaughterhouse using laboratory methods (direct examination following ZiehlHielsen staining and isolation of Mycubacterium strains following culture in a Loewenstein-Jensen medium. During the study, 39 pathogenic Mycobacterium strains (38 Mycobacterium bovis and 1 Mycobacterium tuberculosis) out of 100 sampled out suspicious strains, were studied. No Mycobacteria typical of M. farcinogenes was found on direct examination. We were then able to differentiate between bovine tuberculosis and bovine farcy. The results confirm the well-founded decision in the slaughterhouse to discard the meat on the grounds of tuberculosis. The majority of cattle is located in villages around Bobo-Dioulasso and its is therefore highly likely that the disease is enzootic in the area with a fairly high level of infection in animals.

Key words: Cattle - Tuberculosis - Mycobacterium - Pathogen - Lesion Postmortem examination - Abattoirs - Burkina Faso.
11. MINISTERE DE L'AGRICUL TURE, FT DE L'ELEVAGE, 1993 1994. Rapports annuels statistiques. Bobo Dioulasso, Burkina Faso, Ministère de l'Agriculture et de l'élevage.

12. REY J.L., VILLON A., SALIOU P., 1977. La tuberculose bovine dans le sahel voltaïque. Corrélations avec la tuberculose humaine. Bobo Dioulasso, Hautc-Volta, OCCGE / FAO, 86 p.

13. THOREL M.F., 1994. Le rôle du laboratóire dans le contrôle de la tuberculose chez les animaux. Point vét., 26 (159) : 35-40.

14. TRAORE A., 1994. Etude épidémiologique de la prévalence de la tuberculose bovine à l'abattoir et sur le lait vendu à Bobo-Dioulasso (Burkina Faso). Maisons-Alfort, France, ENVA, 45 p. (Rapport de stage)

15. VESTAL A.L., 1995. Procedures for the isolation of Mycobacteria. Atlanta, Georgia, Centers for Disease Control of the USA, 1969. Atlanta, USA, Public Health Publication.

16. VILLON A., mai 1976. Enquête sur la tuberculose bovine dans le Liptako et l'Oudalan (Département du Sahel, Haute-Volta). Bobo Dioulasso, Haute-Volta, OCCGE. (Doc. technique $\mathrm{N}^{\circ}$ 6357)

DELAFOSSE A., TRAORE A., KONE B. Aislamiento de cepas de micobacterias patógenas en los bovinos sacrificados en el matadero de Bobo-Dioulasso, Burkina Faso. Revue Elev. Méd vét. Pays trop., 1995, 48 (4) : 301-306

La tuberculosis bovina representa el primer motivo de retiro en el matadero de Bobo-Dioulasso. El presente trabajo consistió en confirmar, mediante métodos de laboratorio (examen directo post coloración Ziehl-Nielsen y aislamiento de las cepas de micobacterias después del cultivo sobre un medio de Loewenstein-Jensen), el diagnóstico efectuado mediante necropsia en matadero. Durante la duración de este estudio, se aislaron 39 cepas de micobacterias patógenas (38 cepas de Mycobacterium bovis y one cepa de $M$. tuberculosis), a partir de 100 muestras consideradas sospechosas. Al examen directo no se observó ninguna característica de $M$. farcinogenes. Esto permitió el establecimiento de un diagnóstico diferencial entre la tuberculosis bovina y $\boldsymbol{M}$. farcinogenes. Los resultados confirman los retiros efectuados por tuberculosis en matadero. La mayoria de los bovinos provenían de los pueblos vecinos, situados a proximidad de Bobo-Dioulasso, es possible que esta enfermedad sea enzoótica en la región, con una tasa de infección en los animales muy probablemente elevada.

Palabras clave: Ganado bovino - Tuberculosis - Mycobacterium - Organismo patógeno - Lesión - Inspección postmortem - Matadero - Burkina Faso. 